\title{
Aktuelle Entwicklung und Herausforderungen im Forschungsdatenmanagement in Deutschland
}

\begin{abstract}
Der Artikel beleuchtet relevante aktuelle Entwicklungen in Deutschland auf der Ebene der Nationalen Forschungsdateninfrastruktur und der Bundesländer. Dabei wird auf die damit verbundenen Herausforderungen eingegangen und das Spannungsfeld zwischen lokalen Anforderungen und fachwissenschaftlichen Communities mit internationaler Anbindung betrachtet.
\end{abstract}

\section{Einleitung}

Es ist herausfordernd, bei diesem sehr dynamischen und komplexen Themenfeld überhaupt einen Ausblick zu wagen. Trotzdem soll basierend auf aktuellen Entwicklungen, die die nächsten Jahre - wenn nicht sogar Jahrzehnte - prägen werden, der Versuch unternommen werden, einen Blick in die nahe Zukunft zu werfen und aus heutiger Sicht (Stand August 2020) die mittel- bis langfristigen Herausforderungen zum Abschluss dieses Handbuchs zu skizzieren.

\section{Nationale Forschungsdateninfrastruktur (NFDI)}

Mit dem Beschluss der Gemeinsamen Wissenschaftskonferenz (GWK) im November 2018 hat sich die Bundesrepublik Deutschland das Ziel gesetzt, eine Nationale Forschungsdateninfrastruktur ${ }^{1}$ (NFDI) zu etablieren. Die Diskussion um den Umgang mit Forschungsdaten in einer digital geprägten Wissenschaftswelt hat damit aber weder begonnen noch ihr Ende gefunden; es handelt sich dennoch um einen für das föderale Wissenschaftssystem wichtigen Meilenstein, der den politischen Willen zum gemeinsamen Handeln manifestiert. Die Entwicklung, die sich herausbildenden Strukturen und der aktuelle Stand sollen im Folgenden dargestellt werden.

1 S. https://www.gwk-bonn.de/themen/weitere-arbeitsgebiete/informationsinfrastrukturen-nfdi/. Letztes Abrufdatum der Internet-Dokumente ist der 15.11.2020. 


\subsection{Entwicklung bis zur NFDI}

Die Diskussion um den Umgang mit digitalen Forschungsdaten von der Erhebung bis zur Archivierung und Nachnutzung beinhaltet stets zwei Ebenen. Zum einen können mit Hilfe veröffentlichter Forschungsdaten daraus abgeleitete wissenschaftliche Ergebnisse grundsätzlich auch von anderen verifiziert werden. Zum anderen sind Datenerhebungen, ganz profan gesehen, meist teuer, ethisch problematisch oder nur einmalig möglich, so dass eine Nachnutzung das Verhältnis von Mitteleinsatz zu gesellschaftlichem Nutzen grundsätzlich verbessern kann. Selbstverständlich ist es dafür notwendig, dass der Umgang mit Forschungsdaten dafür - ebenfalls - grundsätzlich und weltweit geregelt werden muss.

Vor dem Hintergrund der damit verbundenen organisatorischen, fachlichen und technischen Herausforderungen gab und gibt es viele weltweite, nationale, regionale und lokale Initiativen und Projekte. Leider kann das Engagement unzähliger Personen hier nur unzureichend gewürdigt werden, aber einige Entwicklungen sollen exemplarisch benannt werden, um zu zeigen vor welchem Hintergrund und in welchem Zusammenhang die NFDI entstand und zukünftig operieren wird. Beispielsweise hat sich 2013 die Research Data Alliance ${ }^{2}$ (RDA) als bottom-up arbeitender, globaler und disziplinübergreifend operierender Zusammenschluss von Institutionen und Mitgliedern gegründet, der sich weltweit in Arbeitsgruppen organisiert und Empfehlungen erarbeitet. Ebenfalls 2013 hat die Deutsche Forschungsgemeinschaft (DFG) das Förderprogramm „Informationsinfrastrukturen für Forschungsdaten ${ }^{3 \text { “ }}$ aufgelegt, 2018 positiv evaluiert und weiterhin als Förderprogramm im Repertoire. Ebenfalls 2013 wurde das Wiki forschungsdaten.org gegründet, welches zusammen mit forschungsdaten.info national bisher Informationen und Initiativen gebündelt hat. 2016 veröffentlichte die Europäische Kommission das Konzept der European Open Science Cloud (EOSC) ${ }^{4}$. Ein Vorschlag für die Umsetzung der EOSC stellt die wiederum bottom-up strukturierte GO FAIR ${ }^{5}$ Initiative dar, die die sogenannten FAIR Prinzipien als Grundsätze für die Wiederverwendbarkeit von Forschungsdaten in den Mittelpunkt stellt. FAIR steht dabei für Findable, Accessible, Interoperable und Reusable. Diese Prinzipien gewährleisten den disziplinen- und länderübergreifenden Zugang zu Daten und ihre Nutzbarmachung.

In einer nationalen Scharnierfunktion zwischen Wissenschaft und Politik hat die GWK - sozusagen top-down in Deutschland - im Jahr 2014 den Rat für Informationsinfrastrukturen $(\mathrm{RfII})^{6}$ eingesetzt. In seiner ersten Amtsperiode hat dieser sich

2 S. https://rd-alliance.org/.

3 S. https://www.dfg.de/foerderung/programme/infrastruktur/lis/lis_foerderangebote/forschungsdaten/.

4 S. https://ec.europa.eu/newsroom/dae/document.cfm?doc_id=15266.

5 S. https://www.go-fair.org/.

6 S. http://www.rfii.de/de/start/. 
intensiv mit dem Thema Forschungsdaten beschäftigt und in seinem ersten Positionspapier $^{7}$ „Leistung aus Vielfalt“ (2016) das Thema aufgearbeitet. Neben einer Analyse liefert das Positionspapier insbesondere auch insgesamt 13 Empfehlungen zu Prozessen, Strukturen und Finanzierung des Forschungsdatenmanagements in Deutschland für alle beteiligten Akteure. Der Rat schlug zur Überwindung der bisherigen fragmentierten und nicht nachhaltig gesicherten Strukturen und Initiativen in Deutschland die NFDI als (dauerhaft) geförderte verteilte nationale Infrastruktur vor, die sich um Abstimmung und Koordination sowie die Verstetigung der projektförmig finanzierten, aber dauerhaft notwendigen Dienste im Bereich des Forschungsdatenmanagements kümmern soll.

Der Aufbau dieser NFDI soll nach Empfehlung des RfII wissenschaftsgetrieben sein und in der Zielvorstellung so den unterschiedlichen Herausforderungen der heterogenen Fachwissenschaften bezogen auf das Forschungsdatenmanagement effizient und doch passgenau begegnen. Damit liegt die NFDI als verteilte, aber vernetzte Infrastruktur von Datenerzeugenden und Datennutzenden quer $\mathrm{zu}$ den bereits bestehenden Säulen im Wissenschaftssystem und soll bundesweit eine vergleichbar gute Versorgung aller datenintensiven Fächer in Bezug auf Forschungsdaten realisieren. Weil Wissenschaft nur global denkbar ist, muss die NFDI gerade trotz des nationalen Auftrags international anschlussfähig sein und als deutschlandweiter starker Knoten der EOSC agieren. „Leistung durch Vielfalt“ und weitere Folgeempfehlungen des RfII wurden nicht nur in Deutschland mit großem Nachhall aufgenommen und haben als Folge dazu geführt, dass am 26.11.2018 von der GWK das neuartige Förderprogramm „NFDI“ per Bund-Länder-Vereinbarung (BLV) geschlossen wurde ${ }^{8}$. Als Rahmenbedingungen wurde das Finanzvolumen von jährlich bis zu 90 Mio. Euro zwischen 2019 und 2028 sowie insgesamt bis zu 30 zu fördernde fachwissenschaftliche Konsortien über drei Ausschreibungsrunden festgelegt. Die Finanzierung wird gemeinsam vom Bund und den Ländern getragen. Der Abschluss dieser Vereinbarung muss als Meilenstein in der Entwicklung im deutschen Wissenschaftssystem und als klares Commitment für eine nationale Aufgabe gesehen werden.

\subsection{Ziele und Struktur der NFDI}

In der BLV zum Aufbau der NFDI wurden auch die Ziele und die Struktur der NFDI ${ }^{9}$ bereits festgelegt. Die GWK folgt inhaltlich damit den Empfehlungen des RfII. Die Durchführung des Ausschreibungs- und Begutachtungsverfahrens entsprechend

7 S. https://d-nb.info/1104292440/34.

8 S. https://www.gwk-bonn.de/fileadmin/Redaktion/Dokumente/Papers/NFDI.pdf.

9 S. https://www.nfdi.de/. 
der Vereinbarung wird in die Hand der DFG gelegt, die Begutachtung folgt den Prinzipien eines wissenschaftsgeleiteten Verfahrens, in dem die Qualität der Anträge vor dem Hintergrund der Ausschreibungskriterien von Forschenden und Infrastrukturbetreibern bewertet wird. Anschließend entscheidet die GWK auf Grundlage der Förderempfehlung der DFG und einem positiven Votum aus dem Begutachtungsverfahren über die Aufnahme von Konsortien in die NFDI. Eine wichtige Rolle im Begutachtungsverfahren spielt das NFDI-Expertengremium ${ }^{10}$, welches in der Logik einer vergleichenden übergeordneten Prüfgruppe die Bewertung aller Anträge auf der Grundlage der fachwissenschaftlichen, informationstechnischen und strukturbezogenen Begutachtung vornimmt sowie die Formulierung der Förderempfehlungen an die GWK formuliert.

Konsortien sind das wissenschaftsbezogene Strukturelement der NFDI. Sie werden in der BLV (und vorher schon vom RfII) definiert:

\begin{abstract}
„Konsortien sind auf langfristige Zusammenarbeit angelegte Zusammenschlüsse von Nutzern und Anbietern von Forschungsdaten wie staatliche und staatlich anerkannte Hochschulen, außeruniversitäre Forschungseinrichtungen, Ressortforschungseinrichtungen, Akademien und andere öffentlich geförderte Informationsinfrastruktureinrichtungen. Sie sind in der Regel nach Fachgruppen bzw. Methoden organisiert, ohne Vorgaben für ihre institutionelle Zusammensetzung. [...] Die Konsortien stellen die Sprech- und Handlungsfähigkeit in der Partnerschaft zwischen wissenschaftlicher Fachgemeinschaft und beteiligten Infrastrukturbetreibern her; sie entwickeln und fördern eine Kultur des Daten-Teilens und der Informationskompetenz gemäß den FAIR-Prinzipien; sie tragen Sorge dafür, dass technische Dienste für die Datenbereitstellung, -archivierung und -erschließung aufgebaut, gepflegt und die dafür notwendigen Datenspeicherungs-und Hardware-Kapazitäten zur Wahrnehmung der nationalen Aufgaben angepasst werden“ ${ }^{11}$
\end{abstract}

Konsortien orientieren sich nach den Empfehlungen des RfII und des NFDI-Expertengremiums der DFG an thematisch-fachlichen Domänen, deren Granularität bewusst nicht festgelegt ist. Sie haben dafür die relevanten Akteure zusammengebunden und sorgen so für Sprechfähigkeit und Normbildung innerhalb der Domäne. Sie sind auf Dauer angelegt, sollen aber dynamisch auf fachliche Anforderungen reagieren können und werden regelmäßig evaluiert. Ihre nachhaltigen Dienste sind ebenfalls nicht einheitlich festgelegt, da sie sich an den Bedarfen ihrer Community ausrichten sollen. Zusätzlich zu der Aufgabe, die Domäne oder Community passgenau abzuholen und für die Zukunft standardisierend zu wirken, haben die Konsortien auch Aufgaben in der NFDI. Alle Konsortien wirken synergetisch zusammen, um generische, über ein Konsortium hinausgehende, sogenannte Querschnittsthemen zu bearbeiten. Bewusst wurden in den bisherigen zwei Ausschreibungsrunden

10 S. https://www.dfg.de/dfg_profil/gremien/gremium/index.jsp?id=426076674.

11 S. BLV-Vereinbarung Paragraph 9 https://www.gwk-bonn.de/fileadmin/Redaktion/Dokumente/ Papers/NFDI.pdf. 
(Stand August 2020) keine Konsortien zu Querschnittsthemen zur Antragstellung aufgefordert.

Die BLV legt als zusätzliche Strukturelemente der NFDI die Konsortialversammlung, den Wissenschaftlichen Senat sowie das Direktorat (angesiedelt am KIT ${ }^{12}$ ) fest, die gemeinsam die NFDI für Deutschland gestalten. Der seit Anfang 2020 eingesetzte Direktor der NFDI hat mit dem Gründungsdirektorat einen Entwurf für die Rechtsform ${ }^{13}$ der NFDI vorgelegt, der von der GWK am 26.06.2020 verabschiedet wurde. Es handelt sich um eine Vereinsstruktur, in der geförderte Konsortien per se Mitglied sind, aber auch andere juristische Personen Mitglied werden können. Zusätzlich zu den Strukturelementen aus der BLV sollen ein Kuratorium und Sektionen etabliert werden. Sektionen dienen der inhaltlichen disziplinübergreifenden $\mathrm{Zu}$ sammenarbeit der sogenannten „Konsortien nach Satzung“ und sollen alle Organe des Vereins insbesondere bei der Vorbereitung der Entscheidungsvorschläge zu konsortienübergreifenden Standards, Metadatenstandards und Formaten unterstützen. Konsortien nach Satzung orientieren sich an den geförderten Konsortien nach der BLV, können aber weitere Vereinsmitglieder integrieren und bieten so einen Ansatz, Querschnittsthemen zu bearbeiten.

Um die notwendige Vernetzung von möglichen Konsortien und die Herausbildung einer NFDI frühzeitig zu unterstützen, hat die DFG bisher in den Jahren $2019^{14}$ und $2020^{15}$ jeweils zu einer NFDI-Konferenz als Vernetzungsformat eingeladen. Dieses Format wurde sehr intensiv und positiv aufgenommen. Das Ziel einer NFDI als verteilte, aber auch vernetzte Struktur, wird auch in dem für die DFG offeneren Begutachtungsverfahren verfolgt. So wird z. B. das Votum der Begutachtungsgruppe den antragstellenden Konsortien so frühzeitig mitgeteilt, dass sie vor der endgültigen Entscheidung im NFDI-Expertengremium noch Stellung zu etwaigen offenen Fragen nehmen können. Trotzdem bleibt es eine Herausforderung, in einem grundsätzlich wettbewerblich orientierten Verfahren bei begrenzten Ressourcen eine gut abgestimmte nationale Gesamtstruktur zu entwickeln. Bei der Breite der NFDI-Konsortien stellt auch die Begutachtung eine Herausforderung dar, bei der bewusst und notwendigerweise auch internationale Expertinnen und Experten eine große Rolle spielen. Diese wiederum sind mit dem föderalen deutschen Wissenschaftssystem mit länderfinanzierten Hochschulen und außeruniversitären Forschungseinrichtungen weniger vertraut.

12 S. https://www.kit.edu/index.php.

13 S. https://cdn.website-editor.net/25abfc2078d74313bbe63818c335df0e/files/uploaded/Satzung\% 2520NFDI\%2520eV_final.pdf.

14 S. https://www.dfg.de/foerderung/programme/nfdi/konferenz_2019/index.html.

15 S. https://www.dfg.de/foerderung/programme/nfdi/konferenz_2020/index.html. 


\subsection{Stand der NFDI}

Zum aktuellen Zeitpunkt (August 2020) ist die erste Förderrunde der NFDI abgeschlossen und die zweite Förderrunde angelaufen. Im Zuge der ersten Förderrunde waren bis zum 15.10.2019 insgesamt 22 Anträge aus insgesamt 142 Einrichtungen eingegangen. ${ }^{16}$ In diesem Überblick der DFG wird festgehalten:

„Die meisten eingegangenen Anträge sind primär den Lebenswissenschaften, die wenigstens den Ingenieurwissenschaften zuzuordnen. Acht der 22 Anträge sind den Lebenswissenschaften zugeordnet (36 Prozent). Sechs Anträge stammen primär aus den Naturwissenschaften (27 Prozent) und fünf Anträge beschreiben primär geistes- und sozialwissenschaftliche Vorhaben (23 Prozent). Lediglich drei Anträge sind primär in den Ingenieurwissenschaften angesiedelt (14 Prozent). Eine nahezu identische fachliche Verteilung findet sich im jährlichen Antragsvolumen nach Wissenschaftsbereich.“

Insgesamt werden in der ersten Runde der NFDI nun neun Konsortien ${ }^{17}$ gefördert. Diese verteilen sich wie folgt auf die Wissenschaftsbereiche ${ }^{18}$ : Vier Konsortien aus den Lebenswissenschaften (45 Prozent), zwei Konsortien aus den Geistes- und Sozialwissenschaftlichen (22 Prozent aller geförderten Konsortien), zwei Konsortien aus den Naturwissenschaften (22 Prozent) und ein Konsortium aus den Ingenieurwissenschaften (11 Prozent).

In dieser ersten Runde hat insbesondere die NFDI-Konferenz im Sommer 2019 strukturbildend gewirkt, waren dort noch insgesamt 57 Konsortien vertreten, so waren es im Juli 2020 bei der zweiten Konferenz ${ }^{19}$ nur noch 39. Davon haben sich einige Konsortien den sogenannten Querschnittsthemen verschrieben, deren Diskussion auf jeder NFDI-Konferenz einen breiten Raum einnahmen, weil es zum jetzigen Zeitpunkt keine Fördermöglichkeiten für Querschnittskonsortien im Rahmen der BLV gibt, diese Themen aber eine wichtige Rolle beim Aufbau spielen.

Zusammengefasst wird der Stand zum jetzigen Zeitpunkt differenziert bewertet: Auf jeden Fall ist es sehr gut und wichtig, dass es in Deutschland das Förderinstrument NFDI gibt. Auch von anderen Ländern wird Deutschland hier als handlungsfähig und richtungsweisend wahrgenommen. Der eingeschlagene Prozess bietet mit dem wissenschaftsgeleiteten Verfahren die Chance, den eingeforderten und notwendigen Kulturwandel bezüglich des Forschungsdatenmanagements tatsächlich und messbar voranzutreiben. Die verteilte und vernetzte Struktur bietet auch die

16 S. https://www.dfg.de/download/pdf/foerderung/programme/nfdi/191212_nfdi_statistik_antragseingang.pdf.

17 S. https://www.dfg.de/foerderung/programme/nfdi/.

18 S. https://www.dfg.de/download/pdf/foerderung/programme/nfdi/20200626_nfdi_foerderentscheidungen.pdf.

19 S. https://www.dfg.de/download/pdf/foerderung/programme/nfdi/nfdi_konferenz_2020/vortrag_eickhoff.pdf. 
Chance, bisherige erfolgreiche Verbünde weiter zu entwickeln. Es bleibt die Herausforderung, die Vielfalt tatsächlich als Leistung und nicht als Hemmschuh zu systematisieren. Ob daraus wirkliche EINE nationale Forschungsdateninfrastruktur entsteht, ist heute $\mathrm{zu}$ hoffen. Die nächsten Monate werden sicherlich mit darüber entscheiden, ob und wie die u.g. Herausforderungen systematisch und erfolgreich adressiert werden können.

\section{Forschungsdatenmanagement in den Bundes- ländern}

Die 16 Bundesländer übernehmen nicht nur eine gestaltende, sondern auch die finanzielle Verantwortung für den Aufbau der NFDI in Deutschland. Dies liegt zum einen darin begründet, dass zahlreiche Bundesländer bereits seit einigen Jahren in die Digitalisierung von Forschung (und Lehre) mit spezifischen Förderprogrammen investieren, zum anderen ist Bildung (im weitesten Sinne) immer noch Länderangelegenheit und damit einhergehende finanzielle Investitionen sichern naturgemäß Wettbewerbsvorteile der eigenen Hochschulen im Bundesland. So mag es auch nicht verwundern, dass drei Bundesländer, die mit ihrer Bewerbung für die Ansiedlung des NFDI-Direktorats in ihrem Bundesland in die Endauswahl gekommen sind, bereits langjährige, millionenschwere Förderprogramme im Vorfeld aufgelegt haben. Konsequenterweise sind dies auch die Bundesländer, die bereits frühzeitig mit eigenen Bundeslandinitiativen zu Forschungsdatenmanagement an den Start gegangen sind.

\subsection{Beispiele für vorbereitende Projekte und Initiativen}

Im Folgenden können nicht alle FDM-relevanten Initiativen und Projekte in den 16 Bundesländern beschrieben werden. Die Auswahl konzentriert sich daher auf besonders einschlägige Bundesländer, die frühzeitig und konsequent über Jahre hinweg in verschiedene Bereiche der Digitalisierung (z. B. Open Access, Forschungsdaten, Lizenzierung, Forschungsdatenrepositorien etc.) mittels dedizierter Förderprogramme investiert haben.

Das Ministerium für Wissenschaft, Forschung und Kunst in Baden-Württemberg veröffentlichte bereits im Mai 2014 die Broschüre „Science - Wissenschaft unter neuen Rahmenbedingungen: Fachkonzept zur Weiterentwicklung der wissenschaftlichen Infrastruktur in Baden-Württemberg ${ }^{20 *}$, die von einer durch das Wissen-

20 S. https://mwk.baden-wuerttemberg.de/de/service/publikation/did/e-science/. 
schaftsministerium eingesetzten Expertenkommission erarbeitet wurde und entlang von sechs Arbeitsgruppen mit den thematischen Schwerpunkten Lizenzierung, Digitalisierung, Open Access, Forschungsdatenmanagement, Virtuelle Forschungsumgebungen strategische Konzepte für jedes Handlungsfeld entwickelt hat. Auf dieser Basis förderte das Ministerium in Baden-Württemberg in den letzten Jahren weitere sog. E-Science-Projekte, die in ihrer Vielfalt und thematischen Schwerpunktsetzung bemerkenswert sind. Dabei verfolgt Baden-Württemberg einen dezentralen und partizipatorischen Ansatz, so dass zahlreiche - wenn nicht alle - Hochschulen in irgendeiner Form an verantwortlicher Stelle aktiv sind und in ein Netzwerk auf Bundeslandebene einbezogen sind.

In Hessen ist insbesondere das Forschungsförderungsprogramm „Landes-Offensive zur Entwicklung Wissenschaftlich-ökonomischer Exzellenz“21 (LOEWE) zu nennen, welches seit 2008 als wissenschaftspolitischer Impulsgeber für die hessische Forschungslandschaft initiiert wurde. Von 2008 bis 2019 standen rund 869 Mio. Euro Landesmittel für die drei Förderlinien (Zentren, Schwerpunkte, KMU-Hochschule-Verbundprojekte) zur Verfügung. Die bisher 15 LOEWE-Zentren und 60 LOEWE-Schwerpunkte wurden von einer eigens eingerichteten LOEWE-Geschäftsstelle im Hessischen Ministerium für Wissenschaft und Kunst administrativ begleitet, wohingegen die 310 Verbundprojekte mit den klein- und mittelständischen Unternehmen (KMU) von der Hessen Agentur GmbH betreut werden. Das Bundesland Hessen verfolgt mit diesem Ansatz in den bisherigen zwölf Förderstaffeln die dezidierte Einbeziehung der KMU. Auch für das Jahr 2020 und folgende Jahre stehen nicht unerhebliche Fördermittel bereit. Allerdings werden im LOEWE-Förderprogramm auch nur in geringem Umfang Vorhaben mit einer Ausrichtung auf E-Science, Open Access etc. gefördert. Nichtsdestotrotz dürfte dieses Programm wesentlich dazu beitragen, dass sich in den letzten Jahren in Hessen kooperative Strukturen herausgebildet haben, die eine partizipatorische Bearbeitung von gemeinsamen Themenschwerpunkten erlauben, wie auch HeFDI (vgl. Kap. 2.2.) zeigt.

In Nordrhein-Westfalen haben sich zu Beginn des Jahres 2017 (bisher) 42 Hochschulen in einer Kooperationsvereinbarung 22 zur „Digitalen Hochschule NRW (DH. NRW)“ ${ }^{23}$ mit dem Ziel zusammengeschlossen, Digitalisierungsprozesse und dafür nötige Maßnahmen im NRW-Hochschulwesen weiterzuentwickeln. Diese Kooperationsvereinbarung berücksichtigt dabei von Beginn an die drei unterschiedlichen Hochschularten in NRW und wurde Ende 2018 mittels einer extra formulierten sog. Verfahrensordnung ${ }^{24}$ organisatorisch und strukturell gestärkt. Darin wird die koope-

21 S. https://wissenschaft.hessen.de/wissenschaft/landesprogramm-loewe.

22 S. https://www.dh.nrw/fileadmin/user_upload/dh-nrw/pdf_word_Dokumente/DH.NRW_Kooperationsvereinbarung.pdf.

23 S. https://www.dh.nrw/.

24 S. https://www.dh.nrw/fileadmin/user_upload/dh-nrw/pdf_word_Dokumente/DH.NRW_Verfahrensordnung.pdf. 
rative Zusammenarbeit der Gremien fixiert. NRW hat im Vergleich zu anderen Bundesländern die Zusammenarbeit der Hochschulen im eigenen Bundesland am stärksten formalisiert und auf eine belastbare Governance-Struktur gestellt. Flankiert werden die Maßnahmen rund um die Digitale Hochschule NRW durch eine Reihe von Förderprojekten, von denen hier nur exemplarisch einige mit Start 2020 genannt werden sollen: „Digitale Werkzeuge für die Hochschullehre“, „E-Drittmittelakte NRW“ oder „Moodle NRW“. Projekte mit Bezug zu FDM sind zum Beispiel FDM-Scouts.nrw explizit für die Fachhochschulen in NRW oder auch Data Literacy Education.nrw. NRW steht als bevölkerungsreichstes Bundesland vor der Herausforderung, eine wesentlich größere Anzahl an Hochschulen mit auf den digitalen Weg zu nehmen und investiert daher entlang zahlreicher Förderprogramme verschiedene Verbundvorhaben mit jeweils einer Hochschule als Konsortialleitung. Eine eigens eingerichtete „Landesinitiative FDM“25 fungiert als „zentrale Koordinierungsstelle, um die Hochschul- und Landesaktivitäten im Kontext von FDM zu bündeln und strategisch weiterzuentwickeln“.

So unterschiedlich die Ausgangslagen in den drei hier exemplarisch beschriebenen Bundesländern bezüglich Anzahl der Hochschulen, Digitalisierungsstrategie auf Bundeslandebene, Engagement im Bereich Forschungsdaten(management) etc. auch sind, so investieren diese Bundesländer nicht unerhebliche Fördermittel, um ihre Hochschulen bei dem dringend benötigten digitalen Kulturwandel und speziell auch im Umgang mit Forschungsdaten zu unterstützen.

\subsection{Bundesländer mit Projekten und Initiativen im Bereich Forschungsdatenmanagement}

Zahlreiche Bundesländer haben mit eigenen, durch ihr jeweiliges Ministerium geförderte, Initiativen zu Forschungsdatenmanagement den ersten Schritt hin zu koordinierenden und vernetzenden Maßnahmen zum Umgang mit Forschungsdaten getätigt. Im Folgenden werden zunächst die Bundesländer vorgestellt, die durch eine dezidierte Förderung eine FDM-Initiative in ihrem Bundesland aufbauen, bevor nachfolgend auch weitere Projekte und Entwicklungen aus den anderen Bundesländern (soweit bekannt) vorgestellt werden.

Die folgenden sechs Bundesländer befinden sich zurzeit (Stand August 2020) in einer Förderphase für den Aufbau bzw. die Weiterentwicklung der eigenen Initiative zu FDM:

25 S. https://www.fdm.nrw/. 


\section{Baden-Württemberg}

Baden-württembergisches Begleit- und Weiterentwicklungsprojekt für Forschungsdatenmanagement (bw2FDM), https://bwfdm.scc.kit.edu/, gefördert vom Ministerium für Wissenschaft, Forschung und Kunst Baden-Württemberg

- Förderzeitraum: nach zwei Vorgänger-Förderphasen seit Mai 2019 bis voraussichtlich April 2023

- Fördervolumen: keine öffentlichen Angaben

Schwerpunkte (exemplarisch):

- $\quad$ Redaktion und Weiterentwicklung von forschungsdaten.info

- Bereitstellen von Workflows, Analysewerkzeugen und Dienste-Portfolios für einzelne Disziplinen

- Informationsvermittlung (Schulungen, Webinare), Beratungen

- Koordinierung und Organisation des Arbeitskreises FDM in BW

- Vernetzung mit den anderen Landesinitiativen

- Koordination der Querschnittsthemen der vier baden-württembergischen Science Data Center

- Planung und Durchführung der Konferenz „E-Science-Tage“

\section{Brandenburg}

Forschungsdatenmanagement in Brandenburg: Technologien, Kompetenzen, Rahmenbedingungen (FDM-BB), http://forschungsdaten-brandenburg.de, gefördert vom Ministerium für Wissenschaft, Forschung und Kultur (MWFK)

- Förderzeitraum: seit November 2019 bis voraussichtlich Dezember 2020, Nachfolgeantrag in Bearbeitung

- Fördervolumen: ca. 110.000 Euro

Schwerpunkte (exemplarisch) ${ }^{26}$

- Aufbau Netzwerk FDM: Acht Hochschulen in Brandenburg, aber auch außeruniversitäre Forschungseinrichtungen, sind in einer Arbeitsgruppe koordiniert und tauschen sich monatlich aus

- Start von Diskussionen im Bereich Institutionalisierung FDM in den Hochschulen unter Einbeziehung der brandenburgischen Landesrektorenkonferenz bzw. Vize-Präsidentinnen und -Präsidenten für Forschung/Entwicklung/Transfer

- Schulungen (bspw. mittels Webinare), Beratung, Unterstützung Forschende etc.

- Identifikation kooperativ zu nutzender Dienste (bspw. FDM-Werkzeug wie RDMO und Treffen von bundeslandweiten Absprachen)

26 Vgl. Wuttke et al. 2020, Schneemann et al. 2020 und Radke et al. 2020. 
- Bedarfs- und Umfeldanalyse für die Formulierung von Handlungs- und Implementierungsempfehlungen an das MWFK mit dem Ziel, eine FDM-Policy für Brandenburg zu entwickeln

- Vernetzung und Austausch mit anderen Bundeslandinitiativen, der NFDI sowie international (z. B. GoFAIR, RDA, EOSC etc.)

\section{Hamburg}

Hamburg Open Science (HOS), https://fdm.hos.tuhh.de/, gefördert von der Freie und Hansestadt Hamburg

- Förderzeitraum: seit Januar 2018 bis voraussichtlich Ende 2020

- Fördervolumen; 3,4 Mio. Euro für Forschungsdaten (für Open Science insgesamt ca. 15 Mio. Euro)

Schwerpunkte (exemplarisch):

- Beratungen, Schulungen

- Aufbau von Repositorien für Forschungsdaten

- Umsetzung der Vorgaben der Forschungsförderer

- Aufbau und Einführung von FDM an den Hamburger Hochschulen

\section{Hessen}

Hessische Forschungsdateninfrastrukturen (HeFDI), https://www.uni-marburg.de/ de/hefdi, gefördert vom Hessisches Ministerium für Wissenschaft und Kunst (HMWK)

- Förderzeitraum: seit Mai 2016 bis voraussichtlich Dezember 2020, Nachfolgeantrag in Bearbeitung

- Fördervolumen: 3,4 Mio. Euro

Schwerpunkte (exemplarisch):

- Dienste und Werkzeuge (z B. Sync \& Share-Dienst via Hessenbox, RDMO-Instanzen für HeFDI-Hochschulen)

- Beratungen, Schulungen (z. B. Forschungsdatenkurse für Studierende, Umgang mit sensiblen Forschungsdaten)

- Mitwirken in Steuerungsgruppe des Projekts Langzeitverfügbarkeit an hessischen Hochschulen (LaVaH)

- Beteiligung DINI/nestor-AG Forschungsdaten, Unterstützung Aufbau NFDI-Konsortien, Unterstützung des Serviceverzeichnis Forschungsdaten, Beteiligung Research Data Alliance (RDA)

- Aufbau FDM-unterstützender Strukturen an allen Standorten mit der Förderung der Zusammenarbeit und des fachlichen Austauschs inklusive des Schaffens von Synergie und Wissenstransfer 


\section{Nordrhein-Westfalen}

Landesinitiative für Forschungsdatenmanagement (fdm.nrw), https://www.fdm. nrw/, gefördert vom Ministerium für Kultur und Wissenschaft

- Förderzeitraum: seit September 2019 bis voraussichtlich Dezember 2021

- Fördervolumen; ca. 1,2 Mio. Euro

Schwerpunkte (exemplarisch):

- Schulungen, Weiterbildung (z. B. Newcomer-Track, Advanced-Track, FDM-Zertifikatskurs)

- Bereitstellung von Tools \& Materialien

- Beratungen (z. B. FDM-Prozess-Begleitung mit Fokus Fachhochschulen)

- Informationsvermittlung und Begleitung von Akteurinnen und Akteuren beim Aufbau der NFDI

- Vernetzung innerhalb des Bundeslands, mit anderen Bundeslandinitiativen und international

\section{Thüringen}

Kompetenznetzwerk Forschungsdatenmanagement der Thüringer Hochschulen (TKFDM), https://forschungsdaten-thueringen.de, gefördert vom Thüringer Ministerium für Wirtschaft, Wissenschaft und Digitale Gesellschaft (TMWWDG)

- Förderzeitraum: von Oktober 2018 bis voraussichtlich Oktober 2021

- Fördervolumen: 600.000 Euro

Schwerpunkte (exemplarisch):

- Materialsammlungen, Best Practice Reihe, Checklisten, Handreichungen

- Thüringer FDM-Tage (Datenpreis-Verleihung)

- Workshops, Schulungen, Coffee Lectures, Webinare

- Beratungen

- Vernetzung innerhalb des Bundeslands, bundeslandübergreifend (z B. DINI/ Nestor) und international (z. B. GO FAIR, RDA)

- Fortschreiben der Digitalisierungsstrategie 2021-2023

\section{Sachsen}

Auch wenn es in Sachsen noch keine explizit geförderte Bundeslandinitiative zu FDM gibt, so scheint Sachsen nach bisherigem Kenntnisstand das nächste, vielversprechende Bundesland mit einer potentiell zukünftigen ministeriellen Förderung zu sein und wird daher hier auch kurz vorgestellt. 
Forschungsdatenmanagement in Sachsen (SaxFDM), https://saxfdm.de/, Förderantrag in Vorbereitung

- Projektlaufzeit ohne Förderung bisher, aber seit 2019 als Bottom-Up-Initiative von Forschungseinrichtungen, Forschenden und unter Beteiligung des Sächsischen Ministeriums für Wissenschaft und Kultur (SMWK)

Schwerpunkte (exemplarisch)

- Etablierung eines Expertennetzwerks für den Aufbau und Betrieb landesweiter Dienste zu verschiedenen Aspekten des FDM

- Schulungen, Weiterbildungen

- Vernetzung innerhalb des Bundeslands und bundesweit (fachliche FDM-Gruppen, RDA-DE, DINI/nestor)

- Langfristiges Ziel: Schaffen einer trag- und zukunftsfähigen Organisationsstruktur einschließlich kooperativem Einsatz von personellen und finanziellen Ressourcen (zentraler Ansprechstelle)

- Strategische Planung der Unterstützung von gemeinsamen Diensten und technischen Infrastrukturen

- Etablierung, Umsetzung und Koordination einer landesweiten Forschungsdatenstrategie

\section{Weitere Ansätze}

Auch in anderen Bundesländern gibt es vielversprechende Ansätze im Bereich FDM, die zum Teil hervorragend als Basis für eine bundeslandweite Initiative dienen können. $\mathrm{Zu}$ nennen sind insbesondere Aktivitäten in den nachfolgenden Bundesländern:

In Bayern fördert das Bayerisches Staatsministerium für Wissenschaft und Kunst (MWK Bayern) seit Januar 2018 bis Dezember 2020 das fachwissenschaftliche Projekt „eHumanities - interdisziplinär“, welches ebenfalls FDM-spezifische Materialien sammelt, Lernmaterialien und -module erstellt und sich innerhalb Bayerns sowie bundesweit (z. B. DHd-Verbund) vernetzt. Im Ergebnis sollen auch Empfehlungen zu FDM in den digitalen Geisteswissenschaften formuliert werden. Als zweites vom MWK Bayern gefördertes Projekt zählt „Digitale Langzeitverfügbarkeit für Wissenschaft und Kultur in Bayern“, welches vom Juni 2019 bis Mai 2021 mit rund 410.000 Euro finanziert ist. Es verfolgt vorrangig die Ziele, LZA-Workflows (dezentral und zentral) zu erstellen, diese technisch umzusetzen, Mustervereinbarung zur digitalen Langzeitarchivierung zu erarbeiten sowie ein nachhaltiges Geschäfts- und Kostenmodells zu entwickeln.

In Berlin existiert an zahlreichen Universitäten seit zum Teil vielen Jahren fundierte Expertise rund um FDM. Im Bereich Open Access werden alle Aktivitäten und Angebote im Open Access Büro Berlin gebündelt, wohingegen dies im Bereich FDM zum jetzigen Zeitpunkt noch nicht sichtbar ist. Ob eine zukünftige Koordinierung 
unter dem Dach der „Berlin University Alliance“ angesiedelt wird, durch eine thematische Öffnung des Open Access Büros Berlin passiert oder alternative Szenarien entwickelt werden, scheint noch unklar zu sein.

In Bremen hat sich insbesondere die Universität Bremen mit dem im Jahr 2019 vom RatSWD akkreditiertem Forschungsdatenzentrum (FDZ) Qualiservice um FDM verdient gemacht.

In Mecklenburg-Vorpommern hat sich an der Universität Rostock die dortige Universitätsbibliothek des Themas FDM angenommen und bietet umfangreiche Unterstützungen inklusive einer ausführlichen FAQ-Liste etc. an.

In Niedersachen sind bereits seit einigen Jahren zahlreiche Hochschulen sehr aktiv im Bereich Forschungsdatenmanagement. Exemplarisch seien hier die TIB Hannover (Leibniz-Informationszentrum Technik und Naturwissenschaften und Universitätsbibliothek) mit ihrem Engagement in $\mathrm{DOI}^{27}$ genannt oder die Georg-August-Universität Göttingen mit der eResearch Alliance ${ }^{28}$. Zu Beginn des Jahres 2020 hat die wissenschaftliche Kommission in Niedersachsen $\left(\mathrm{WKN}^{29}\right)$ eine Arbeitsgruppe zum Thema FDM eingerichtet, die beratend Empfehlungen für Niedersachsen erarbeiten soll.

In Rheinland-Pfalz existieren gleich an mehreren Hochschulen bzw. in einem Verbund (z.B. Katholische Hochschule Mainz, Johannes Gutenberg-Universität Mainz, Servicezentrum eScience, Technische Universität Kaiserslautern) vielversprechende Ansätze zu FDM, die allerdings bisher auf Bundesland-Ebene noch nicht koordiniert bzw. vernetzt zu sein scheinen.

In Schleswig-Holstein bringt sich die Christian-Albrechts-Universität zu Kiel nicht nur bereits seit Jahren aktiv in FDM-Themen ein und stellt umfangreiche Materialien auf ihren Seiten zur Verfügung, sondern hat auch als eine der ersten Universitäten in Deutschland bereits im Jahr 2015 eine „Leitlinie zum Umgang mit For-

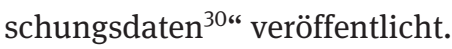

Die Bibliothek der Universität des Saarlandes ist ebenfalls seit Jahren aktiv im FDM-Kontext unterwegs. So hat die Universität des Saarlandes bereits 2001 folgenden Grundsatz zu Forschungsdaten beschlossen: „Primärdaten, die als Grundlage für Veröffentlichungen dienen, sind in derjenigen Einrichtung (Labor, Fachrich-

27 S. https://www.tib.eu/de/publizieren-archivieren/doi-service.

28 S. https://www.eresearch.uni-goettingen.de/de/.

29 Die Wissenschaftliche Kommission in Niedersachsen (WKN) ist ein unabhängiges Expertengremium, das 1997 auf Kabinettsbeschluss dauerhaft eingerichtet wurde und das die niedersächsische Landesregierung in Fragen der Wissenschafts- und Forschungspolitik berät, vgl. www.wk.niedersachsen.de.

30 S. http://www.uni-kiel.de/download/pm/2015/2015-408-leitlinie-forschungsdaten.pdf. 
tung, Klinik), in der sie entstanden sind, für zehn Jahre aufzubewahren, soweit dies zum Zweck der Nachprüfbarkeit notwendig ist. ${ }^{31 \text { “ }}$

In Sachsen-Anhalt ist die Martin-Luther-Universität Halle-Wittenberg sowohl fachspezifisch mit dem „Historischen Datenzentrum Sachsen-Anhalt ${ }^{32 \text { “ }}$ als auch Universitätsweit im Rahmen eines Expertennetzwerks zu Forschungsdaten aktiv, bei dem Vertreter verschiedener Fachbereiche fachspezifische Bedarfe zentral abstimmen.

\subsection{Bundesländer mit Projekten und Initiativen im Bereich For- schungsdatenmanagement}

Die Mehrheit der 16 Bundesländer hat sich auf den langen Weg gemacht, Forschungsdatenmanagement auch auf Ebene der einzelnen Hochschulen zu institutionalisieren. Dabei werden verschiedene Ansätze in der Umsetzung, thematischen Schwerpunktsetzung und unterschiedliche Grade in der Formalisierung erkennbar. Nordrhein-Westfalen scheint hier insgesamt mit der Kooperationsvereinbarung und den zahlreichen flankierenden Maßnahmen sehr weit $\mathrm{zu}$ sein. $\mathrm{Zu}$ beobachten ist auch ein West-Ost-Gefälle: Während in den sieben östlichen Bundesländern bisher nur zwei relativ junge Bundesland-Initiativen gestartet sind, sind diese im Vergleich zu den westlichen FDM-Initiativen finanziell auch deutlich schlechter ausgestattet. Und auch wenn sich bw2FDM in Baden-Württemberg mit forschungsdaten.info um eine Anlaufstelle mit einem Redaktionsteam, in dem alle Bundesländer vertreten sind, organisatorisch und inhaltlich verantwortlich zeigt, ist der Grad der Vernetzung der Bundesland-Initiativen untereinander noch nicht wirklich weit vorangeschritten. Absprachen über gemeinsam zu entwickelnde Schulungsmaterialien, kooperativ erstellte Informationssammlungen etc. sind nicht zu beobachten.

Naturgemäß liegen die Schwerpunkte vieler Initiativen und v. a. einzelner Projekte in den Bundesländern ohne eigene finanzierte FDM-Initiative auf den Bedarfen der eigenen Hochschulen in dem jeweiligen Bundesland. Auch haben einige Bundesländer nur Universitäten und nicht auch Fachhochschulen bzw. Hochschulen der Angewandten Wissenschaften etc. im Fokus. Viele Bundeslandinitiativen konzentrieren sich thematisch in erster Linie auf Qualifizierungsmaßnahmen „vor Ort“, d. h. zum Beispiel um Schulungen sowohl der Forschenden als auch Forschungsunterstützenden in zentralen Informationsinfrastruktureinrichtungen wie Bibliothek oder Rechenzentrum. Nicht viele Bundesland-Initiativen scheinen bisher mit HPCAnwendern vernetzt zu sein oder die Kooperation zum Beispiel mit der Gauss-Alli-

31 S. https://www.uni-saarland.de/fileadmin/upload/verwaltung/fundstellen/Forschungsangelegenheiten/DB01-342.pdf.

32 S. https://www.geschichte.uni-halle.de/struktur/hist-data/. 
$\mathrm{anz}^{33} \mathrm{zu}$ suchen, obwohl gerade diese Forschergruppen naturgemäß Unmengen an Daten produzieren bzw. verarbeiten.

Festzuhalten bleibt, dass den Bundesland-Initiativen und auch den Einrichtungen in den Bundesländern ohne eigene FDM-Initiative eine Schlüsselrolle zukommt, um die Bewusstseinsbildung und Akzeptanz sowie die dringend benötigte Qualifizierung aller Beteiligter rund um Forschungsdaten in die Fläche bis hin zu der kleinen Hochschule in eher abgelegenen Regionen voranzutreiben. Nur so können die NFDI und die EOSC auf ein breites Fundament gestellt werden. Und nur mit Hilfe der Bundesländer können auch Forschende für das Thema FDM sensibilisiert werden, die sich fachwissenschaftlich perspektivisch keiner der 30 NFDI-Fachkonsortien anschließen können.

\section{Zukünftige Herausforderungen}

Wie immer bei dem Start großer Initiativen und Förderprogrammen - allein die NFDI wird im nächsten Jahrzehnt fast eine Milliarde Euro kosten - scheinen die Herausforderungen und Risiken zu überwiegen. Nichtdestotrotz sind mit Entwicklungen dieser Art auch sehr große Chancen verbunden und können im Fall von Deutschland tatsächlich einen kulturellen Wandel im Umgang mit digitalen Daten bewirken. Im Folgenden werden mehrere Herausforderungen und mögliche Lösungsansätze sowie offene Fragen beleuchtet.

Es wird weiterhin für alle Akteurinnen und Akteure notwendig sein, sich immer wieder klar zu machen, dass ein systematisches Forschungsdatenmanagement kein Selbstzweck ist, sondern für den Forschungsstandort Deutschland, aber auch für jeden einzelnen Forschenden zur guten wissenschaftlichen Praxis gehört. Die strukturierte und standardisierte Beschreibung von Daten(sammlungen) muss selbstverständlicher Teil des wissenschaftlichen Arbeitens werden und optimalerweise wird die Veröffentlichung von Daten ein wesentliches Qualitätskriterium zukünftiger Berufungsverfahren bei Professorinnen und Professoren. Alle Forschenden und Studierenden müssen quasi beim Betreten der akademischen Welt auf selbstverständliche Art und Weise im Umgang mit digitalen Daten geschult werden, es muss also Teil ihrer akademischen DNA werden.

Dies stellt u.a. die Hochschulen vor enorme Herausforderungen: Quasi jeder Studiengang muss mindestens ergänzt werden um Module, die FDM zu Thema haben. Gänzlich neue Curricula müssen schnellstmöglich mit definierten Abschlüssen (z. B. data steward etc.) entwickelt werden, die international anschlussfähig sind. Es darf nicht vergessen werden, dass es von der Entwicklung bis hin zu ersten Absol-

33 S. https://gauss-allianz.de/. 
ventinnen und Absolventen mit den neuen Kompetenzen und Fähigkeiten mindestens sechs Jahre vergehen. Es müssen aber nicht nur neue Studiengänge entwickelt und etabliert, sondern auch neue Berufsfelder definiert werden. Im Moment scheint völlig unklar zu sein, welcher Kompetenzkanon den Begriffen wie z. B. data scientist, data analyst, data engineer, data manager, data curator, data librarian, data steward etc. zugrunde liegt, wie sie sich unterscheiden, wie die Karrierepfade aussehen und wie deutsche Berufsbezeichnungen entwickelt werden können etc.

Akademische Ausbildung ist die eine Seite der Medaille, Schulung und Weiterqualifizierung stellt die andere Seite dar. Wie können die unzähligen Mitarbeitenden, die bereits in Lohn und Brot stehen, systematisch geschult werden? Und was sind überhaupt die FDM-relevanten Kerninhalte pro Berufszweig? Was ist erwartbar und realistisch? Wer bezahlt dies? Wie können seriöse und bestenfalls zertifizierte Maßnahmen identifiziert werden? Werden sogenannte Zertifikatskurse, angeboten von Hochschulen, eine zusätzliche Einnahmequelle für Hochschulen darstellen?

Eine weitere Ebene der Herausforderungen betrifft das Verhältnis NFDI zu den Initiativen und Entwicklungen in den Bundesländern. Naturgemäß hat die NFDI die Fach-Konsortien im Blick und fördert die Communities, die besonders strukturbildend in ihrem Fach auftreten. Aber wieviel Prozent der (Nachwuchs-)Wissenschaftlerinnen und Wissenschaftler erreicht der durch die NFDI ausgelöste kulturelle Wandel? Werden die geförderten NFDI-Fachkonsortien im schlimmsten Fall nur als Leuchtturmprojekte wahrgenommen, die außerhalb der jeweiligen Fachdisziplin Niemanden sonderlich interessieren werden? Oder ist es wirklich realistisch anzunehmen, dass z. B. vielleicht zwei bis vier geförderte geisteswissenschaftliche Fachkonsortien tatsächlich hunderttausende geisteswissenschaftlich Studierende zu dem gewünschten, ja fast geforderten Paradigmenwechsel einladen?

Hier kommen die Bundesländer ins Spiel, die quasi vor Ort für ihre Hochschulen in ihrem Bundesland aktiv werden und z. T. große Fördersummen investieren, damit einzelne Forschende, aber auch Studierende und Forschungsunterstützende im Bereich FDM beraten, unterstützt und geschult werden. Es macht weder Sinn noch scheint es überhaupt ansatzweise realistisch zu sein, dass Fachkonsortien Strahlkraft auf einzelne Hochschulen in der Fläche haben werden und in der Lage sind, alle Fakultäten und Fachbereiche einer Hochschule $\mathrm{zu}$ inspirieren. Damit kommt den Bundesländern eine besondere Verantwortung zu und sie müssen ihre Hochschulen überzeugen, FDM als Teil der ureigenen Aufgabe zu verstehen und diese bei den nächsten Hochschulabschlüssen (weitestgehend) aus dem Grundetat zu finanzieren. Eine undankbare Aufgabe, und während sich „Ruhm und Ehre“ auf die leuchtenden NFDI-Fachkonsortien verteilen, müssen sich die Hochschulen und Forschungseinrichtungen im Zweifel in der Fläche allein abmühen. Dadurch entsteht auf ganz natürliche Weise das Spannungsfeld zwischen Exzellenz in der fachwissenschaftlichen Spitze und dem mühsamen Locken und Werben in der Breite bzw. Fläche. Es wird spannend sein zu sehen, ob und wie sich alle Bundesländer in 
koordinierter und systematischer Weise in den NFDI-Prozess ${ }^{34}$ einbringen. In diesem Zusammenhang scheint es auch sehr wichtig zu sein, alle 16 Bundesländer gleichermaßen „ins Boot zu holen“ und auch insbesondere die östlichen Bundesländer verstärkt zu motivieren, sich des Themas FDM anzunehmen. Sowohl politisch als auch finanziell ist hier zwischen West und Ost bereits eine Schieflage zu beobachten. Auch die Zusammenarbeit zwischen außeruniversitären Forschungseinrichtungen und den länderfinanzierten Hochschulen innerhalb der NFDI wird sich weiter ausdifferenzieren müssen.

Eine weitere Herausforderung besteht in dem Verhältnis fachwissenschaftlicher FDM-Expertise auf der einen Seite und die Verantwortung für die sogenannten Querschnittsaufgaben auf der anderen Seite. Natürlich haben sich die jetzt geförderten NFDI-Fachkonsortien dazu verpflichtet, Querschnittsaufgaben wahrzunehmen und sich mit anderen Konsortien entsprechend in z. B. Arbeitsgruppen und Sektionen zu vernetzen. Es bleibt abzuwarten, ob hier ein Schub, um nicht zu sagen, Ruck durch alle Fachdisziplinen erreicht wird und sich die Fachkonsortien verantwortlich fühlen, diesen eher generisch anzulegenden Prozess unter Berücksichtigung aller Beteiligten, also auch derjenigen ohne NFDI-Förderprojekt, an verantwortlicher Stelle zu koordinieren. Erfahrungsgemäß sind diese Prozesse sehr langwierig, beschwerlich und (leider) wenig karrierefördernd. Es wird spannend sein zu beobachten, wer sich in welcher Form für welchen Querschnitts-Themenbereich einsetzen wird, wie dies im NFDI-Ökosystem angesehen wird und welche Auswirkungen sich außerhalb der NFDI ergeben. Immerhin liegt mit der „Leipzig-Berlin-Erklärung zu NFDI-Querschnittsthemen der Infrastrukturentwicklung“35 ein zwischen den Querschnittsinitiativen und (potenziellen) Fachkonsortien abgestimmter Fahrplan vor.

Zusammenfassend kann festgehalten werden, dass das NFDI-Ökosystem sowohl horizontal als auch vertikal kein festes Gefüge darstellen kann, sondern im partizipatorischen Aushandlungsprozess, v. a. auch unter Berücksichtigung der nicht an erster Stelle Beteiligten, inkludierend, offen, neugierig und sich agil verändernd auftreten sollte. Und selbstverständlich muss dieser NFDI-Organismus europäisch und international fest verankert sein und in beide Richtungen (nach Deutschland hinein und aus Deutschland hinaus) transparent informieren, unterstützen, Überzeugungsarbeit leisten etc.

Zum Schluss soll zudem noch auf eine Herausforderung der besonderen Art hingewiesen werden. Natürlich spricht die NFDI mit den im Kielwasser strömenden Hype-Worten wie HPC, KI, Algorithmen, „Blech“36 etc. besonders den Sprach- und Gedankenschatz der männlich Involvierten an und beflügelt offenbar insbesondere Männer, die zu einem erstaunlich großen Prozentsatz den NFDI-Konsortien vorste-

34 An dieser Stelle ist nicht der GWK-Beschluss zur Förderung von NFDI-Fachkonsortien gemeint. 35 Vgl. Bierwirth et al. 2020.

36 Originalzitat auf der 2. NFDI/DFK Konferenz im Juli 2020. 
hen. Selbst in den geisteswissenschaftlichen NFDI-Konsortien (gefördert in der 1. Runde bzw. einzureichen in der 2. Runde) ist der Frauenanteil derer, die im Rahmen der 2. NFDI/DFG-Konferenz präsentiert haben, verschwindend gering.

Auch ist die kulturelle Vielfalt in der bisherigen NFDI-Welt nicht besonders ausgeprägt, dies betrifft sowohl den kulturellen Hintergrund, unterschiedliche Hochschultypen oder auch die Altersstruktur der sogenannten Meinungsführerschaft.

\section{Literatur}

Letztes Abrufdatum der Internet-Dokumente ist der 15.11.2020.

Bierwirth, Maik, Frank Oliver Glöckner, Christian Grimm, Sonja Schimmler, Franziska Boehm, Christian Busse, Andreas Degkwitz, Oliver Koepler und Heike Neuroth. 2020. „Leipzig-BerlinErklärung zu NFDI-Querschnittsthemen der Infrastrukturentwicklung.“ Zenodo. doi:10.5281/ zenodo.3895208.

Radtke, Ina, Niklas Hartmann, Heike Neuroth, Laura Rothfritz, Ulrike Wuttke, Janine Straka, Miriam Zeunert und Carsten Schneemann. 2020. „Anforderungserhebung bei den brandenburgischen Hochschulen.“Zenodo. doi:10.25932/publishup-48091.

Schneemann, Carsten, Miriam Zeunert, Laura Rothfritz, Heike Neuroth, Niklas Hartmann und Ina Radtke. 2020. „Rahmendaten zu FDM-Bundeslandinitiativen.“Zenodo. doi:10.5281/zenodo.4068170.

Wuttke, Ulrike, Heike Neuroth, Laura Rothfritz, Janine Straka, Miriam Zeunert, Carsten Schneemann, Niklas Hartmann, Ina Radtke. 2020. „Umfeldanalyse zum Aufbau einer neuen Datenkultur in Brandenburg (FDM-BB).“ Zenodo. doi:10.25932/publishup-48090. 
